\title{
Stochastic Computational Fluid Mechanics
}

\author{
Stochastic simulations in computational fluid dynamics let researchers model uncertainties \\ beyond numerical discretization errors. The authors present examples of stochastic \\ simulations of compressible and incompressible flows and provide analytical solutions for \\ verifying these newly emerging methods for stochastic modeling.
}

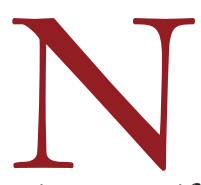

umerical solution of differential equations with stochastic inputs has direct impact on simulations of flow systems in at least three areas: uncertainty quantification, stability of noisy systems, and coarse-grained and multiscale representation. In the first category, the past few years have seen an increasing interest in uncertainty quantification in large-scale numerical simulations. In simulations, just as in experiments, we often question our results' accuracy and construct error bounds a posteriori, but the new objective is to model uncertainty from the simulation's start, not to do it simply as an afterthought. Researchers have used numerical accuracy and error control in flow simulations for some time now, at least in the more modern discretizations, but there's still an uncertainty component associated with the physical problem (specifically, with diverse factors such as constitutive laws, boundary and initial conditions, transport coefficients, source and interaction terms, geometric irregularities, and so on).

$1521-9615 / 07 / \$ 25.00 \odot 2007$ IEEE

Copublished by the IEEE CS and the AIP

Guang Lin, Xiaoliang Wan, Chau-Hsing Su, AND GEORGE EM KARNIADAKIS

Brown University
We encounter noisy nonlinear systems in many applications, from the nanoscale (as in self-assembly processes) to the macroscale (such as large, sudden, disturbances in flow past an aircraft). Bifurcations and chaotic transitions in stochastic dynamical systems can differ greatly from instabilities in deterministic dynamical systems. ${ }^{1}$ How exactly extrinsic stochasticity interacts with intrinsic stochasticity caused by the systems' nonlinear interactions is an intriguing and not-well-understood problem. In turbulent boundary layers, in which a wide range of small scales exists, the mean flow seems to be totally unaffected, even in the presence of substantial background turbulence. ${ }^{2}$ In contrast, for other flow systems at a low Reynolds number, even small amounts of noise can have a significant effect on the mean flow's structure. ${ }^{3}$

The third area in the aforementioned list involves complex systems with an extremely large number of degrees of freedom-models of turbulent flows at a very high Reynolds number or atomistic simulations of mesoscopic size systems. Frequently, we use a coarse-grained procedure to remove degrees of freedom making relatively small contributions to the overall system's energy. The removed degrees of freedom are usually accounted for by stochastic terms in the dimensionally reduced "effective" or "upscaled" equations. In the dissipative particle dynamics method, ${ }^{4}$ for example, coarse-graining of the molecular dynamics method 


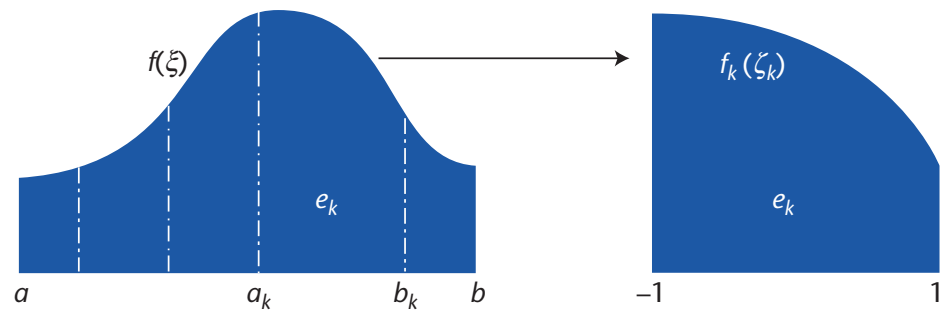

Figure 1. Schematic of decomposition and mapping in multielement generalized polynomial chaos (ME-gPC). The procedure is similar to the deterministic finite element method.

leads to a system of stochastic ordinary differential equations (ODEs) that must be solved efficiently for a very large number of particles.

In this article, we present an efficient nonstatistical approach for modeling extrinsic stochasticity for both compressible and incompressible flows. We consider two prototype cases-flow past a wedge and flow past a bluff body - and quantify uncertainties associated with inflow disturbances, random motions, and random geometric roughness. This is a new field, so properly verifying the results is particularly important. To this end, we've derived analytical solutions for small disturbances based on stochastic perturbation analysis, and we employ these solutions in the verification procedure. Validation of stochastic computational fluid dynamics (CFD) models is a much more difficult task because it requires very accurate characterization experimentally of both inputs and outputs, which is currently lacking in existing data.

\section{Polynomial Chaos and Its Variants}

Polynomial chaos represents a stochastic process by a spectral expansion based on Hermite orthogonal polynomials in terms of Gaussian random variables. Roger G. Ghanem and Pol D. Spanos pioneered its use in solving stochastic differential equations by employing a Galerkin projection to derive an equivalent system of deterministic equations; this can, typically, be solved with standard numerical techniques. ${ }^{5}$ Dongbin Xiu and George E. Karniadakis developed generalized polynomial chaos (gPC), which uses a broader family of trial bases and is based on the orthogonal polynomials from the Askey scheme. ${ }^{6}$ As an example, gPC can express a general second-order random process $T(\omega)$ as

$$
T(\omega)=\sum_{i=0}^{\infty} \hat{T}_{i} \phi_{i}(\xi(\omega))
$$

where $\omega$ is the random event, and the family $\left\{\phi_{i}\right\}$ is an orthogonal basis in terms of the random vector $\xi(\omega)$ with the following orthogonality relation

$\left\langle\phi_{i}, \phi_{j}\right\rangle=\left\langle\phi_{i}^{2}\right\rangle \delta_{i j}$

where $\delta_{i j}$ is the Kronecker delta, and \langle\rangle denotes the ensemble average with respect to the probability density function (PDF) of $\xi$. For a certain random vector $\xi$, we can choose the gPC basis $\left\{\phi_{i}\right\}$ in such a way that its weight function has the same form as the PDF of $\xi$. Gaussian random variables, for example, are associated with Hermite polynomials, uniform random variables are associated with Legendre polynomials, and so forth.

Global gPC expansions work effectively for many physical applications, but increasing the polynomial order might not be efficient for some problems, such as those with random frequencies or low regularity in the parametric space. Based on gPC, Xiaoliang Wan and Karniadakis developed the multi-element extension (ME-gPC), which decomposes the random space into finite elements as in the deterministic finite element method. ${ }^{7}$ Figure 1 illustrates this idea in the one-dimensional case. We assume that the range $[a, b]$ of the $1 \mathrm{D}$ random variable $\xi$ is decomposed into elements $e_{k}$ $:=\left[a_{k}, b_{k}\right]$. We then define a new random variable $\zeta_{k}, k=1,2, \ldots, N$, in each random element, $e_{k}$, as

$\xi=\frac{b_{k}-a_{k}}{2} \zeta_{k}+\frac{b_{k}+a_{k}}{2}$,

with a re-scaled PDF

$$
f_{k}\left(\zeta_{k}\right)=\frac{f\left(\xi\left(\zeta_{k}\right)\right)}{\operatorname{Pr}\left(\xi \in e_{k}\right)} \frac{b_{k}-a_{k}}{2}, \quad k=1, \ldots, N,
$$

where $N$ is the number of random elements, $f(\xi)$ is the PDF of $\xi$, and

$\operatorname{Pr}\left(\xi \in e_{k}\right)=\int_{e_{k}} f(\xi) d \xi$

is the probability that $\xi$ is located in random element $e_{k}$. We first approximate the desired random field $u(\xi)$ locally via gPC, where the degree of perturbation is effectively decreased by Equation 2 from $O(1)$ to $\left.O\left(\left(b_{k}-a_{k}\right) / 2\right)\right)$. Subsequently, we gather the information from all random elements to obtain the statistics of $u(\xi)$.

Because the PDF of $\xi$ is also decomposed together with the random space, gPC's orthogonality in the entire random space will be, in general, lost in random elements. However, given an arbitrary PDF, we can construct the following orthog- 
onal system numerically on the fly, from the threeterm recurrence relationship

$$
\begin{aligned}
\pi_{i+1}\left(\zeta_{k}\right) & =\left(\zeta_{k}-\alpha_{i}\right) \pi_{i}\left(\zeta_{k}\right)-\beta_{i} \pi_{i-1}\left(\zeta_{k}\right), i=0,1, \ldots \\
\pi_{0}\left(\zeta_{k}\right) & =1, \pi_{-1}\left(\zeta_{k}\right)=0
\end{aligned}
$$

where $\left\{\pi_{i}\left(\zeta_{k}\right)\right\}$ is a set of (monic) orthogonal polynomials,

$\pi_{i}\left(\zeta_{k}\right)=+$ lower-degree terms, $i=0,1, \ldots$,

and the coefficients $\alpha_{i}$ and $\beta_{i}$ are uniquely determined by a positive (probability) measure $f_{k}\left(\zeta_{k}\right)$. The orthogonal system $\left\{\pi_{i}\left(\zeta_{k}\right)\right\}$ will then serve as a local gPC basis. For problems related to low regularity in the parametric space, we use the ME-gPC method adaptively to achieve high efficiency. ${ }^{8}$

\section{Galerkin versus Collocation Projection}

For simplicity and clarity, we consider the stochastic equation $L(\boldsymbol{x}, t, \xi(\omega) ; u)=f(\boldsymbol{x}, t ; \xi(\omega))$ with a general (nonlinear) differential operator $L$, where $x \in$ $\mathbb{R}^{d}, d=1,2,3$, indicates the physical space and $t$ the time. In the Galerkin formulation, we first apply the $\mathrm{gPC}$ expansion to

$u(x, t ; \xi(\omega))=\Sigma_{i=0}^{P-1} u_{i} \phi_{i}$

and

$f(x, t ; \xi(\omega))=\Sigma_{i=0}^{P-1} f_{i} \phi_{i}$.

Here $P$ is the total number of basis modes. Then, performing the Galerkin projection on both sides of the equation, we obtain

$$
\left\langle L\left(\boldsymbol{x}, t, \xi(\omega) ; \sum_{i=0}^{P-1} u_{i} \phi_{i}\right), \phi_{k}\right\rangle=\left\langle f, \phi_{k}\right\rangle,
$$

where $k=0,1, \ldots, P-1$. In contrast, in the collocation formulation, we employ Delta functions $\delta\left(\xi-\xi_{k}\right)$ as test functions, $k=0, \ldots, M-1$, where $\left\{\xi_{k}\right\}$ is a proper set of grid (quadrature) points on the range of $\xi(\omega)$, and $M$ is the number of grid points. By applying collocation projection on both sides of the equation, we obtain

$L\left(x, t, \xi_{k} ; u\right)=f\left(x, t ; \xi_{k}\right)$.

Obviously, Equation 4 shares the same format as the original equation, whereas Equation 3 is different. In particular, if the operator $L$ is nonlinear, Equation 3 is a system of ordinary or partial differ- ential equations with the unknowns $u_{i}$ coupled together, which introduces a real challenge to design an efficient deterministic solver. For complex fluid dynamical systems, such as compressible flows, it's easier to use the collocation projection to obtain the governing equations.

Note that the stochastic collocation method is equivalent to solving a deterministic problem at each grid point. Once we obtain the numerical solutions at all collocation points, we can evaluate the random solution's statistics using the corresponding quadrature rule, for example,

$$
\mathbb{E}[u](\boldsymbol{x}, t)=\int u(\boldsymbol{x}, t, \xi) f(\xi) d \xi \approx \sum_{k=0}^{M-1} u\left(\boldsymbol{x}, t, \xi_{k}\right) w_{k},
$$

where $w_{k}$ represents the integration weights, and $\mathbb{E}$ denotes the expectation.

\section{High Dimensionality and Sparse Grids}

Xiu and Jan S. Hesthaven ${ }^{9}$ constructed a stochastic collocation method based on sparse grids by using the Smolyak algorithm. ${ }^{10}$ This algorithm is a linear combination of tensor product formulas, and the resulting grid set has a significantly smaller number of grids compared to the full tensor product rule. Sparse grids depend weakly on the random space's dimensionality, and hence are more suitable for applications with large dimensional random inputs. Recently, sparse grids for high-dimensional integrals have also received a lot of attention. ${ }^{11-13}$

\section{Representation of Stochastic Inputs}

We represent the stochastic inputs with the Karhunen-Loeve (KL) decomposition ${ }^{14}$ given by

$v(x ; \omega)=\bar{v}(x)+\sum_{i=1}^{\infty} \sqrt{\lambda_{i}} \psi_{i}(x) \xi_{i}(\omega)$,

where $v(x ; \omega)$ denotes the random process, $\bar{v}(\boldsymbol{x})$ denotes the corresponding mean, $\left\{\xi_{i}(\omega)\right\}$ is a set of uncorrelated random variables with zero mean and unit variance, and $x$ is the spatial or temporal coordinate. Also, $\psi_{i}(\boldsymbol{x})$ and $\lambda_{i}$ are the eigenfunctions and eigenvalues of the covariance kernel $R_{b b}(x, y)$, respectively-that is,

$\int_{D} R_{b b}(x, y) \psi_{i}(y) d y=\lambda_{i} \psi_{i}(x)$.

Specifically, for a time-dependent stochastic process, we usually consider the exponential covariance kernel 


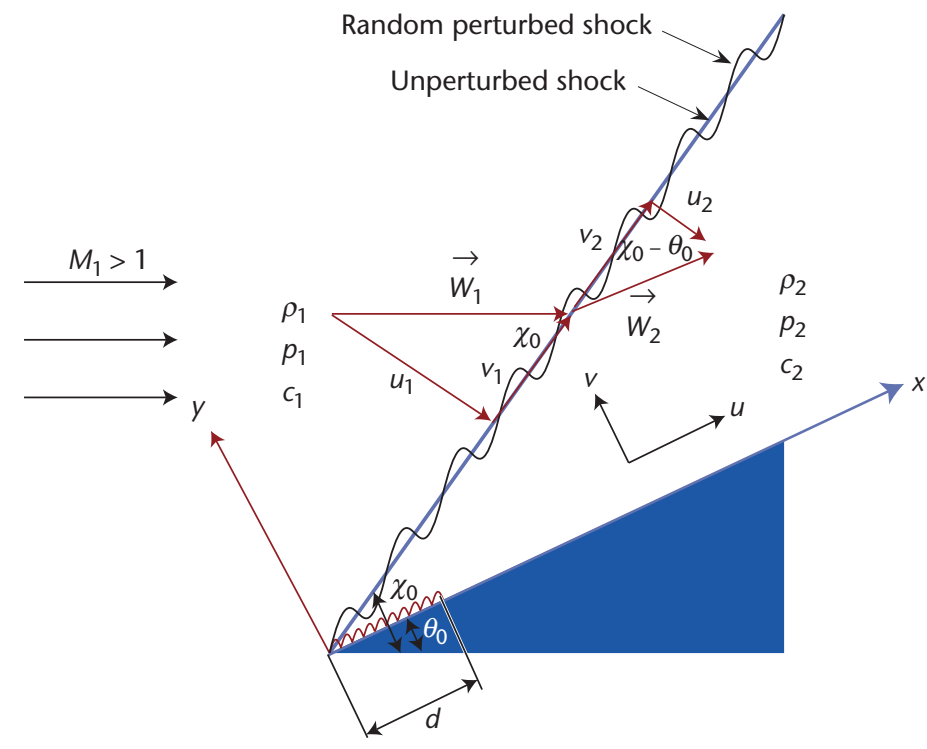

Figure 2. Sketch of supersonic flow past a wedge and definition of a coordinate system. We assume two different random disturbances corresponding to random inflow velocity or random oscillations of the wedge around its apex. We're interested in investigating how such random disturbances affect the shock path.

$$
\begin{aligned}
& R_{b h}\left(v\left(t_{1}, \omega\right), v\left(t_{2}, \omega\right)\right) \\
& \quad=\mathbb{E}\left[v\left(t_{1}, \omega\right) v\left(t_{2}, \omega\right)\right]=e^{\frac{-\left|t_{1}-t_{2}\right|}{A}},
\end{aligned}
$$

where $A$ is the correlation length. This corresponds to a first-order Markov chain in time, which in discrete form is

$v_{0}=\xi_{0}, v_{i+1}=c v_{i}+q \xi_{i+1}, \quad i \geq 0$

where $c=e^{\frac{-\Delta t}{A}}, q=\sqrt{1-c^{2}}$, and $\Delta t$ is the time step.

Discrete forms of stationary spatial-dependent random processes usually differ from time-dependent ones. This is due to the time series' unilateral nature, which only depends on past values as opposed to the spatial process's dependence in all directions. By solving the following stochastic Helmholtz equation, we can obtain the corresponding spatial covariance kernel $R_{b b}\left(v\left(\boldsymbol{x}_{1}, \omega\right), v\left(\boldsymbol{x}_{2}, \omega\right)\right){ }^{15}$

$$
\Delta v-k^{2} v=f(x)
$$

where the random forcing term $f(x)$ is a whitenoise process, a function of the spatial vector $x$ that satisfies

$$
\mathbb{E}\left[f\left(x_{1}\right) f\left(x_{2}\right)\right]=\delta\left(x_{1}-x_{2}\right) .
$$

Equation 9 corresponds to a discrete model for a second-order autoregressive process in space, where the value of $v$ at $x$ depends on its neighbors. Here's a simple 1D example:

$v_{i}=\frac{c}{2}\left(v_{i+1}+v_{i-1}\right)+a \xi_{i}$.

The parameter $c$ is the constant correlation coefficient, $a$ gives the measure of the stochastic forcing's strength, and $\xi_{i}$ are independent random variables with zero mean and unit variance.

\section{Stochastic Simulations}

Let's first look at some results for compressible inviscid steady flows and then for incompressible unsteady flows.

\section{Stochastic Compressible Flows}

For supersonic flows, random inflow disturbances can substantially modify the flow field, thus rendering traditional predictive CFD tools invalid. We study the shock dynamics in two-dimensional supersonic flows past a wedge, assuming two different random disturbances. These correspond to random inflow velocity or random oscillations of the wedge around its apex, and they can be steady in time or time-dependent (the latter could be a model for aeroelastic motions, such as flutter oscillations). Figure 2 shows a schematic. We consider small perturbations and assume that the shock slope's perturbation is small. We approximate the flow between the shock and the wedge as isentropic.

We denote the wedge angle by $\theta_{0}$, the shock angle by $\chi_{0}$, and the incoming flow velocity $W_{1}$ with its normal component $u_{1}=W_{1} \sin \chi_{0}$. The streamlines behind the shock are parallel to the wedge surface, and we denote the velocity by $W_{2}$ and its normal component to the shock by $u_{2}=W_{2} \sin \left(\chi_{0}-\right.$ $\left.\theta_{0}\right)=W_{1} \cos \chi_{0} \tan \left(\chi_{0}-\theta_{0}\right)$.

First, we assume that the wedge angle is fixed, but the shock is subject to random inflow perturbation that we describe as a uniform random variable. We've obtained an analytical solution for the perturbed shock path

$$
z(x ; \xi)=x\left(1+s^{2}\right) \frac{\Delta M_{1}}{H\left(\chi_{0}\right)},
$$

where $s=\tan \left(\chi_{0}-\theta_{0}\right), \Delta M_{1}$ is the perturbed part of the inflow Mach number, and $H\left(\chi_{0}\right)=d M_{1} / d \chi$. The mean and variance of the perturbed shock path are then

$\langle z(x ; \xi)\rangle=0$ 


$$
\begin{aligned}
\operatorname{Var}(z(x ; \xi)) & =x^{2}\left(1+s^{2}\right)^{2} \frac{M_{1}^{2} \varepsilon^{2}\left\langle\xi^{2}\right\rangle}{H^{2}\left(\chi_{0}\right)} \\
& =x^{2}\left(1+s^{2}\right)^{2} \frac{M_{1}^{2} \varepsilon^{2}}{3 H^{2}\left(\chi_{0}\right)} .
\end{aligned}
$$

Next, we assume that the wedge inflow is deterministic and describe the wedge oscillations as a uniform random variable. In this case, we obtain

$$
z(x ; \xi)=-x\left(1+s^{2}\right) \frac{M_{1} \varepsilon \xi}{H\left(\chi_{0}\right)} .
$$

The mean and variance for this case are similar to the previous case, consistent with physical intuition.

Numerically, we solve the 2D Euler equations following the polynomial chaos approach for random inflow and random oscillations. In the latter case, we use a transformation based on a boundaryfitted coordinate system approach, so that we solve the Euler equations in a stationary domain:

$u_{t}+f(u)_{x}+g(u)_{y}=T(u)$,

where $u=[\rho, m, n, E]^{T}, f=[\rho u, u m+p, u n, u(p+E)]^{T}$, and $g=[\rho v, v m, v n+p, v(p+E)]^{T}$; for random inflow, $T=0$, whereas for random oscillations, $T=[0$, $\left.-\rho \cos \theta_{0}\left(u_{w}\right)_{t}, \rho \sin \theta_{0}\left(u_{w}\right)_{t}, \rho\left(u_{w}\right)_{t}\left(n \sin \theta_{0}-m \cos \theta_{0}\right)\right]^{T}$. Here, $u_{w}$ represents the wedge's random motion.

In these examples, we consider the case in which the inflow velocity is perturbed by random fluctuation, which we describe as a random variable with amplitude $\varepsilon=0.01$ and $\varepsilon=0.18$. Figure 3 presents the mean of density $\rho(x, y ; \xi)$ corresponding to inflow perturbation described as a random variable with amplitude $\varepsilon=0.18$. We note that the mean resembles a fan expansion. Figure 4 plots the perturbed shock path's variance as a function of the distance from the wedge apex $x$ on the wedge surface for two amplitude values: $\varepsilon=0.01$ and $\varepsilon=0.18$. From Equation 13, we know that the perturbed shock's variance is proportional to $x^{2}$. Indeed, for amplitude $\varepsilon=0.01$, we can verify that the perturbation solution from Equation 13 and the numerical solution for amplitude $\varepsilon=0.01$ match exactly. However, for amplitude $\varepsilon=0.18$, we see in Figure 4 that the numerical solution deviates from the analytical solution in Equation 13 because that equation doesn't hold for large amplitudes.

\section{Stochastic Wedge Roughness}

Next, let's consider the perturbation of an oblique attached shock in supersonic flow past a wedge due to random roughness on the wedge's surface. ${ }^{16}$ Figure 2 defines the problem, and we use the same as-

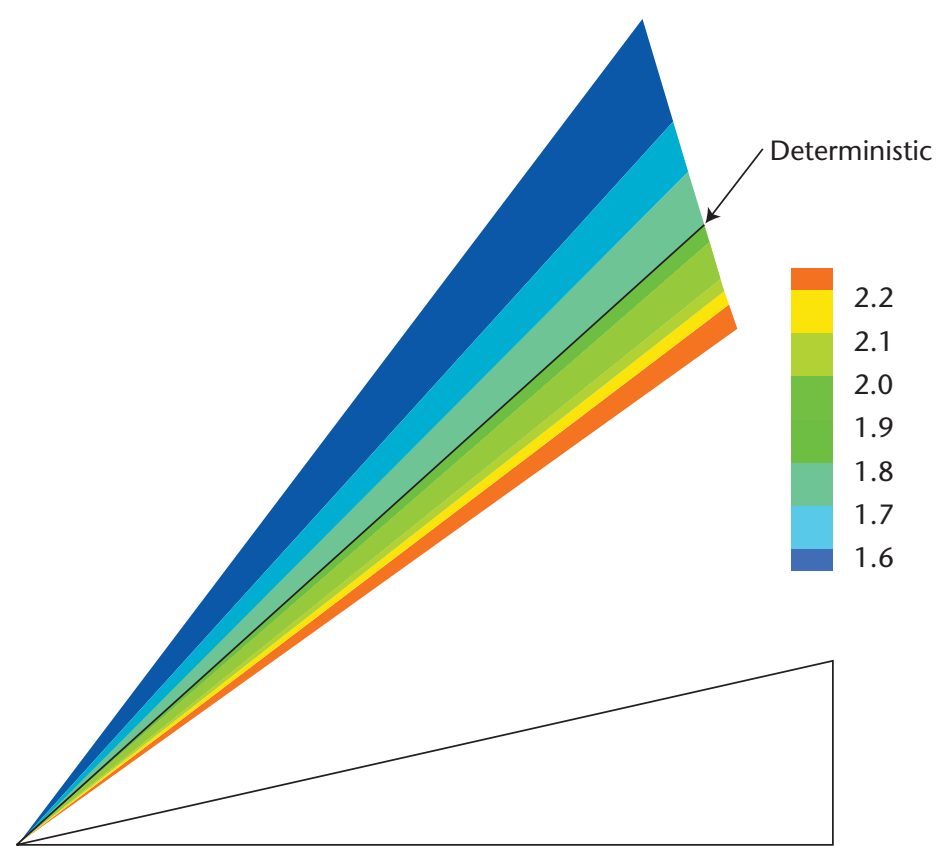

Figure 3. Mean of $\rho(x, y ; \xi)$ induced by random inflow perturbation described as a random variable with amplitude, $\varepsilon=0.18$. Note that the mean resembles a fan expansion.

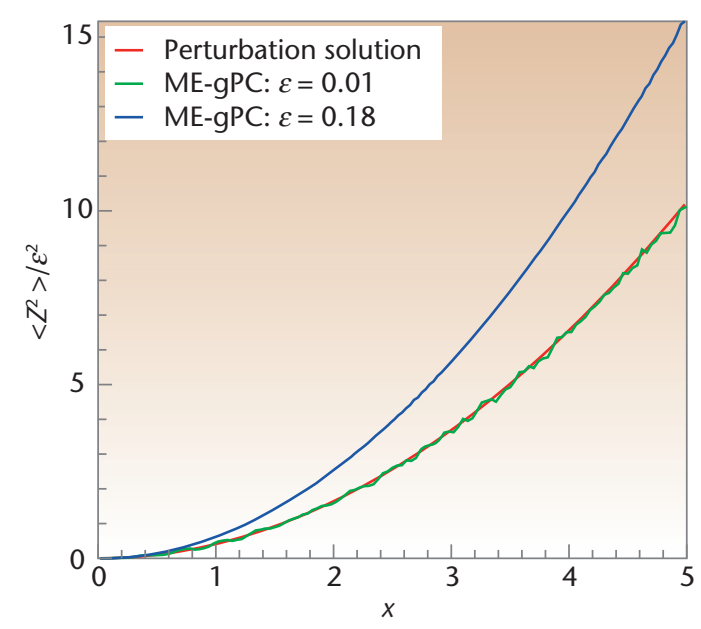

Figure 4. Variance of perturbed shock paths as a function of $x$ induced by random inflow perturbation, which is described as a random variable with amplitude $\varepsilon=0.01$ and $\varepsilon=0.18$. The perturbed shock path's variance obtained from the numerical simulations for amplitude $\varepsilon$ $=0.01$ is proportional to $x^{2}$, which matches with the perturbation solution. However, for amplitude $\varepsilon=0.18$, the numerical solution deviates from the analytical solution because the perturbation analysis doesn't hold for large amplitude. 


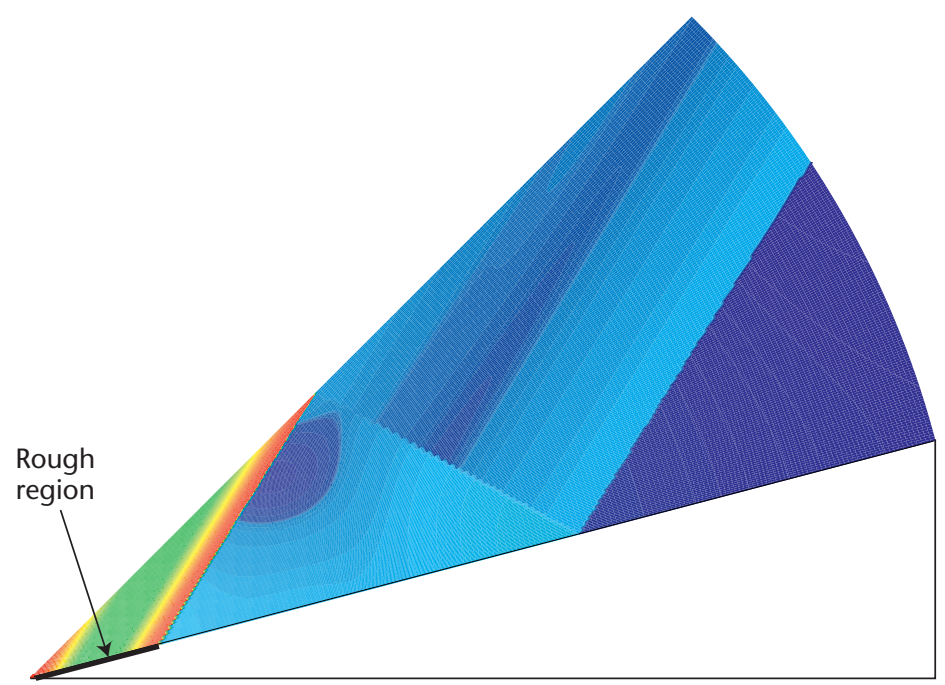

Figure 5. Contours of the normalized standard deviation of the perturbed pressure $\sigma(p) / e$. Red denotes high values, and blue denotes lower values.

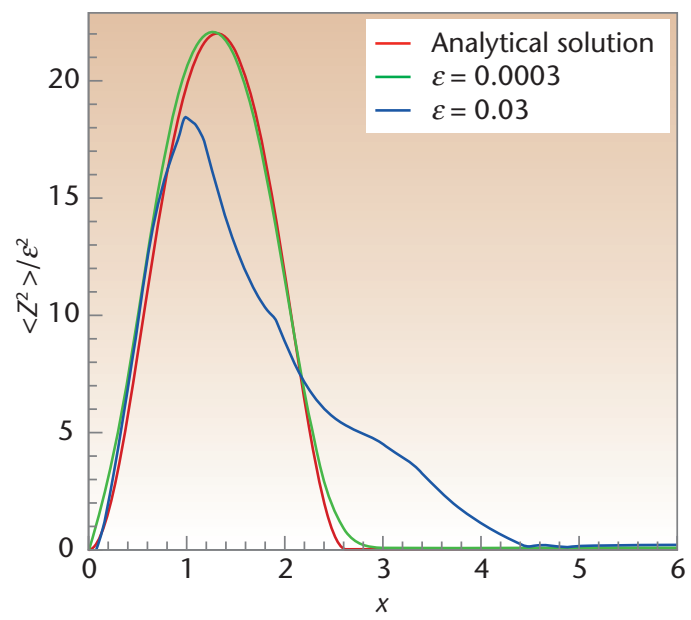

Figure 6. Variance of the perturbed shock path as a function of $x$. The random roughness is described as a random process with correlation length $A / L=1$.

sumptions as in the previous case.

Using stochastic perturbation analysis, we obtain the perturbed shock path $z(x ; \xi)$ as

$z(x ; \xi)=\varepsilon q\left(1+s^{2}\right) \sum_{n=0}^{\infty}(-r)^{n} \frac{h\left(\alpha \beta^{n} x ; \xi\right)}{\alpha \beta^{n}}$

where $\varepsilon h(x)$ is the perturbation on the wedge and $q\left(\chi_{0}\right)=\frac{2}{F\left(\chi_{0}\right)+G\left(\chi_{0}\right)}$,
$r\left(\chi_{0}\right)=\frac{F\left(\chi_{0}\right)-G\left(\chi_{0}\right)}{F\left(\chi_{0}\right)+G\left(\chi_{0}\right)}$,

$\alpha=1-s / m$, and

$\beta=\frac{m-s}{m+s}$.

Both $\alpha$ and $\beta$ are less than 1 ; we obtain $M_{2}, F\left(\chi_{0}\right)=$ $d \theta / d \chi$, and

$G\left(\chi_{0}\right)=\frac{\sqrt{M_{2}^{2}-1}}{\gamma M_{2}^{2} P_{2}} \frac{d P_{2}}{d \chi}$

from normal shock relations.

We assume the length of the wedge is finite $(x \in$ $[0, L])$ and describe the perturbation at the rough wedge $y(x ; \xi)=\varepsilon b(x ; \xi)$ as a space-dependent random process, described as a second-order autoregressive process between $[0, L]$ with zero mean and exponential covariance: ${ }^{15}$

$$
\left\{\begin{array}{l}
h_{i}=\frac{b}{2}\left(b_{i-1}+b_{i+1}\right)+a \xi_{i}, \quad i=1,2, \ldots, N-1 \\
b=e^{-\frac{\Delta x^{2}}{2 A^{2}}}, \quad a=-0.5(\Delta x)^{\frac{3}{2}} e^{-\frac{\Delta x^{2}}{2 A^{2}}},
\end{array}\right.
$$

where $A$ is the correlation length in space. We set the rough perturbation at $x=0$ and $L$ to be zero $\left(b_{0}=b_{N}=0\right)$.

Numerically, we solve the 2D Euler equations in Equation 15 with $T=0$, following the collocation approach. Because it's a steady-state problem, we solve Equation 15 by marching in time until the flow reaches a steady state. Figure 5 shows the contours for the normalized standard deviation of the perturbed pressure $\sigma(p) / e$. Figure 6 presents the variance of the perturbed shock as a function of $x$. We describe the random roughness as a random process with correlation length $A / L=1$; for amplitude $\varepsilon=0.0003$, we can verify that the analytical solution from Equation 16 and the numerical solution match exactly. However, for the larger amplitude $\varepsilon=0.03$, we see in Figure 6 that the numerical solution loses its symmetry and deviates substantially from the analytical solution obtained from Equation 16.

\section{Noisy Flow Past a}

\section{Stationary Circular Cylinder}

Let's consider a model problem that appears in many engineering applications: noisy flow past a 
stationary circular cylinder. The inflow velocity $u=$ $U(t)$ is random, which Figure 7 indicates with right arrows that have rippled ends. The 2D incompressible Navier-Stokes equations govern the flow:

$$
\begin{aligned}
& \frac{\partial u_{i}}{\partial t}+u_{j} \frac{\partial u_{i}}{\partial x_{j}}=-\frac{\partial p}{\partial x_{i}}+\frac{1}{\mathbb{E}[\operatorname{Re}]} \frac{\partial^{2} u_{i}}{\partial x_{j}^{2}}, \quad i=1,2 \\
& \frac{\partial u_{j}}{\partial x_{j}}=0 \text { (summation over } j \text { ). }
\end{aligned}
$$

We normalize all length scales with the cylinder diameter $D$, all velocity components with the mean inflow velocity $\mathbb{E}[U]$, and the pressure with $\rho \mathbb{E}^{2}[U]$ (where $\rho$ is the fluid density). We define the Reynolds number as $\operatorname{Re}=U D / v$, where $v$ is the fluid's kinematic viscosity.

For a deterministic uniform flow past a stationary circular cylinder, we can model the lift force $F_{L}$ on the cylinder as harmonic in time at the shedding frequency $f_{s}$ :

$$
F_{L}(t)=\frac{1}{2} \rho U^{2} D C_{L}^{d} \cos \left(2 \pi f_{s} t\right),
$$

where $C_{L}^{d}$ is the maximum amplitude of the instantaneous deterministic lift coefficient. We want to extend such a harmonic model to the stochastic case. If the inflow velocity $u=U(t ; \xi)$ is random, the corresponding lift force and vortex shedding frequency will also be random. We can express the instantaneous stochastic harmonic model of $C_{L}$ as

$$
C_{L}(t)=A_{L}(\xi) \cos \left(2 \pi f_{s}(\xi) t\right)
$$

and rewrite $f_{s}(\xi)$ as

$$
f_{x}(\xi)=\mathbb{E}\left[f_{s}\right]+\sigma_{f_{s}} X
$$

where $\sigma_{f}$ is the standard deviation of $f_{s}$ and $X \in[a$, $b]$ is a random variable with unit variance. We then obtain the following statistics of $C_{L}$ :

$$
\begin{array}{ll}
\mathbb{E}\left[C_{L}\right] \rightarrow 0, & \text { as } t \rightarrow \infty, \\
\mathbb{E}\left[C_{L}^{2}\right] \rightarrow \frac{1}{2} \int_{a}^{b} A_{L}(\xi(x)) f(x) d x, & \text { as } t \rightarrow \infty,
\end{array}
$$

where $f(x)$ is the PDF of $X$. We note that the second-order moment approaches a constant value for an arbitrary random variable $\xi$ in contrast to the oscillatory behavior for the deterministic case.

Let's consider the following boundary condition at the inflow:

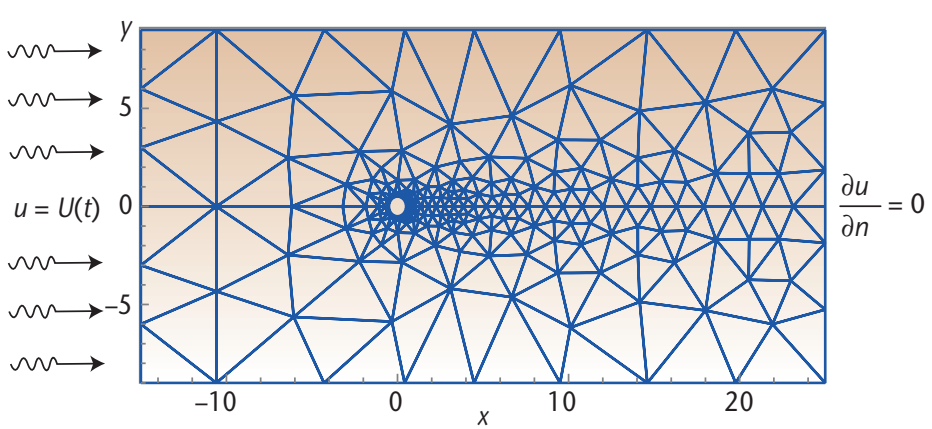

Figure 7. Schematic of the domain and mesh for noisy flow past a circular cylinder. The size of the domain is $[-15 D, 25 D] \times[-9 D, 9 D]$, and the cylinder is at the origin with diameter $D=1$. The mesh consists of $\mathbf{4 1 2}$ triangular elements.

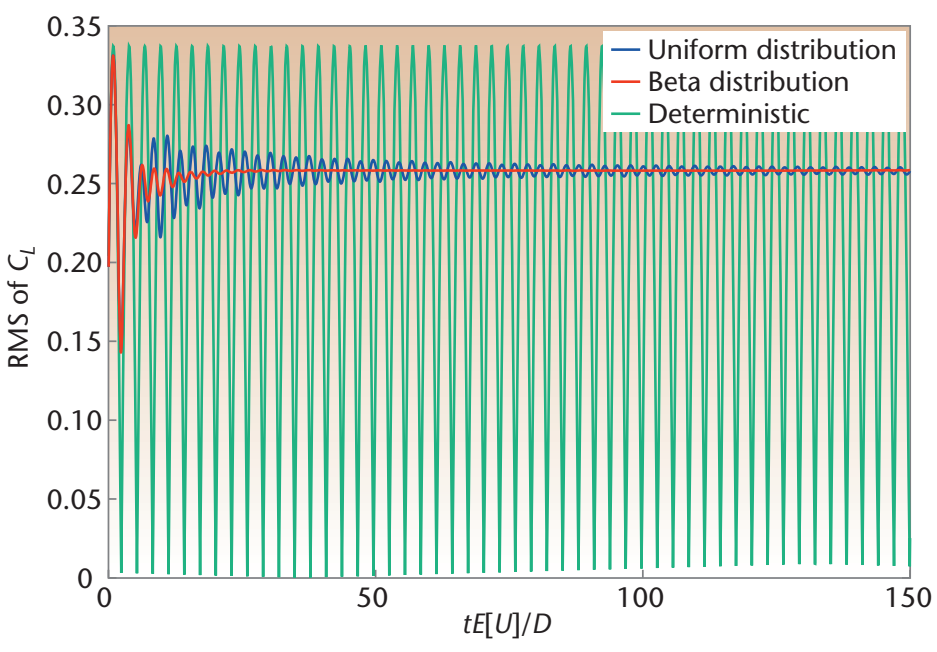

Figure 8. Instantaneous root-mean-square (RMS) of lift coefficients. A uniform distribution in $[-\sqrt{3}, \sqrt{3}]$ and a beta distribution $\operatorname{Be}(2,2)$ in $[-\sqrt{7}, \sqrt{7}]$ are considered with $\sigma=0.1$.

$u=1+\sigma \xi, \quad v=0$

where $\xi$ is a random variable with zero mean and unit variance, and $\sigma$ is a constant indicating the degree of perturbation. We let $\mathbb{E}[\operatorname{Re}]=100$. For 10 percent symmetric noise $(\sigma=0.1)$, the range of the Reynolds number is $\operatorname{Re} \in[90,110]$. Using an empirical relation between the vortex-shedding frequency and the Reynolds number, ${ }^{17}$ we find that the corresponding vortex-shedding frequency is random: $f_{s} \in[0.1592,0.1697]$. We've shown that the random frequency can severely weaken gPC's efficiency in long-term integration. ${ }^{18} \mathrm{gPC}$ 's accuracy with polynomial order $p=15$, for example, will reach an error of $O(1)$ at $t \mathbb{E}[U] / D \approx 40$ for this case. To simulate 10 percent symmetric noise (uniform 

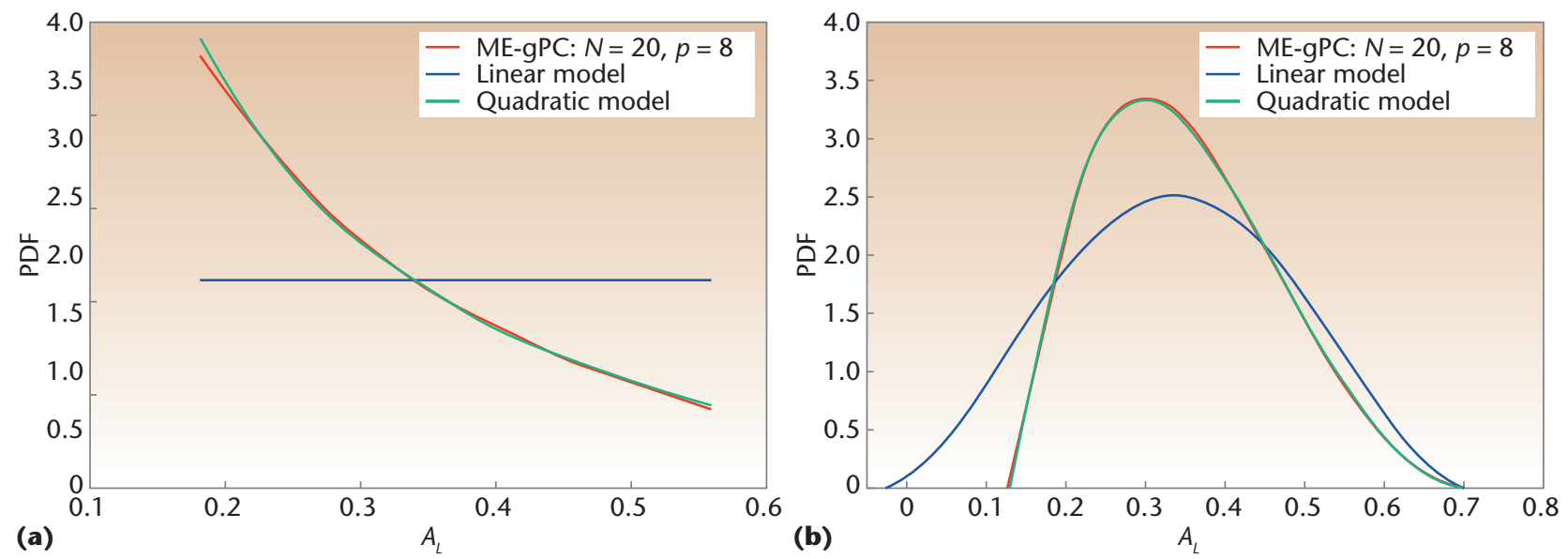

Figure 9. Probability density functions (PDFs) of $A_{L}$ given by different models: (a) uniform noise and (b) beta noise. The relation between $\xi$ and $A_{L}$ is well described with a quadratic model.

and beta PDFs), we use ME-gPC with $N=20$ and $p=8$, which can reach an accuracy of $O\left(10^{-3}\right)$ in the range $t \mathbb{E}[U] / D \leq 150$, corresponding to roughly 20 shedding periods. We can see in Figure 8 that the root-mean-square (RMS) of lift coefficient approaches a constant value quickly, in agreement with the stochastic analytic model of $C_{L}$. The RMS approaches approximately the same value, 0.258 , for both uniform and beta noise, which is 20 percent bigger than the time-averaged deterministic RMS, or 0.215 , without noise.

Subsequently, we study the random amplitude $A_{L}$ by incorporating a linear model, $A_{L}(\xi)=A_{0}+$ $c_{1} \xi$, and a quadratic model, $A_{L}(\xi)=A_{0}+c_{2} \xi+c_{3} \xi^{2}$, where $A_{0}=0.3372$ is the deterministic amplitude at $\mathrm{Re}=100$ given by direct numerical simulations, and $c_{i}, i=1,2,3$ are undetermined constants. Using the first- and second-order moments, we can resolve $c_{i}$ from the ME-gPC results. For the uniform noise, we obtain $c_{i}=0.1402,0.1100$, and 0.0109; for the beta noise, $c_{i}=0.1414,0.1101$, and 0.0112 . These two sets of numbers agree well because the linear and quadratic models are independent of the PDF of $\xi$. We observe from the linear model that the response degree is $c_{1} / A_{0} \approx 42$ percent in contrast to the 10 percent noise at the inflow, which implies that the lift coefficient is very sensitive to upstream noise. Due to the quadratic term, the RMS of $C_{L}$ is bigger than the deterministic one at $\mathrm{Re}=100$ as Figure 8 illustrates. Figure 9 shows the PDFs of $A_{L}$. We see that they're well described by the quadratic model, which is consistent with the deterministic model of lift coefficient. ${ }^{17}$ In contrast to symmetric noise, the PDF of $A_{L}$ has a clear bias toward lower Reynolds numbers.

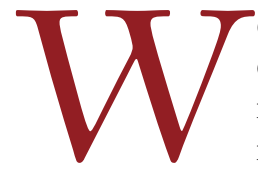

e're at the dawning of the stochastic modeling age in fluid mechanics, which means there's much more work to be done. The computational complexity of a typical stochastic simulation is more than an order of magnitude greater than its deterministic counterpart, but parallel algorithms are rather straightforward to implement and can significantly reduce the required simulation time. Establishing "error bars" in CFD that reflect not only numerical uncertainty but also uncertainty in physical modeling and geometry provides great credibility to simulation and will make it an indispensable tool in designing complex flow systems. It's an important step in establishing simulation-based certificates of fidelity in flow design. In addition, stochasticsimulated responses can serve as a form of sensitivity analysis that could potentially guide experimental work and dynamic instrumentation and make the simulation-experiment interaction more meaningful.

Several outstanding issues related to long-time integration, stochastic discontinuities, adaptivity, and high dimensionality still exist. All of them are key elements to establishing multi-element gPC (with Galerkin or collocation projections) as a "mainstream" stochastic simulation approach and a powerful alternative to Monte Carlo simulation. These methods are more suitable for unsteady simulations and several orders of magnitude more efficient than MC for the stochastic correlated inputs typically encountered in CFD applications.

\section{Acknowledgments}

This work was supported by the US Air Force Office of 
Scientific Research's computational mathematics program and the US National Science Foundation's AMC-SS program.

\section{References}

1. E. Simiu, Chaotic Transition in Deterministic and Stochastic Systems, Princeton Univ. Press, 2002.

2. P.K. Maciejewski and R.J. Moffat, "Heat Transfer with Very High Free-Stream Turbulence, Part I: Experimental Data," I. Heat Transfer, vol. 114, no. 44, 1992, pp. 827-833.

3. D. Lucor and G.E. Karniadakis, "Noisy Inflows Cause a SheddingMode Switching in Flow Past an Oscillating Cylinder," Physical Rev. Letters, vol. 92, no. 15, 2004, pp. 154501(1-4).

4. P. Espanol and P. Warren, "Statistical Mechanics of Dissipative Particle Dynamics," Europhysics Letters, vol. 30, no. 4, 1995, pp. 191-196.

5. R.G. Ghanem and P. Spanos, Stochastic Finite Elements: A Spectral Approach, Springer-Verlag, 1991.

6. D. Xiu and G.E. Karniadakis, "The Wiener-Askey Polynomial Chaos for Stochastic Differential Equations," SIAM J. Scientific Computing, vol. 24, no. 2, 2002, pp. 619-644.

7. X. Wan and G.E. Karniadakis, "Multi-Element Generalized Polynomial Chaos for Arbitrary Probability Measures," SIAM J. Scientific Computing, vol. 28, no. 3, 2006, pp. 901-928.

8. X. Wan and G.E. Karniadakis, "An Adaptive Multi-Element Generalized Polynomial Chaos Method for Stochastic Differential Equations," J. Computational Physics, vol. 209, no. 2, 2005, pp. 617-642.

9. D. Xiu and J.S. Hesthaven, "High Order Collocation Methods for Differential Equations with Random Inputs," SIAM J. Scientific Computing, vol. 27, no. 3, 2005, pp. 111-113.

10. S. Smolyak, "Quadrature and Interpolation Formulas for Tensor Products of Certain Classes of Functions," Soviet Mathematics Doklady, vol. 4, 1963, pp. 240-243.

11. E. Novak and K. Ritter, "High Dimensional Integration of Smooth Functions over Cubes," Numerische Mathematick, vol. 75, no. 11, 1996, pp. 79-97.

12. G. Wasilkovski and H. Wozniakowski, "Explicit Cost Bounds of Algorithms for Multivariate Tensor Product Problems," J. Complexity, vol. 11, no. 1, 1995, pp. 1-56.

13. T. Gerstner and M. Griebel, "Numerical Integration Using Sparse Grids," Numerical Algorithms, vol. 18, nos. 3 and 4, 1998, pp. 209-232.

14. M. Loeve, Probability Theory, 4th ed., Springer-Verlag, 1977.

15. C.H. Su and D. Lucor, "Covariance Kernel Representations of Multidimensional Second-Order Stochastic Processes," J. Computational Physics, vol. 217, no. 1, 2006, pp. 82-99.

16. G. Lin, C.H. Su, and G.E. Karniadakis, "Modeling Random Roughness in Supersonic Flow past a Wedge," Proc. AlAA Aerospace Sciences Meeting and Exhibit, AIAA Press, 2006, pp. 2006-0124.

17. R. D. Blevins, Flow-Induced Vibration, Van Nostrand Reinhold Company, 1990.

18. X. Wan and G.E. Karniadakis, "Long-Term Behavior of Polynomial Chaos in Stochastic Flow Simulations," Computational Methods in Applied Mathematics \& Eng., vol. 195, nos. 41-43, 2006, pp. 5582-5596.

Guang Lin is a PhD candidate in the Division of Applied Mathematics at Brown University. His research interests include scientific computation, uncertainty quantification, and MHD flow simulations. Lin has an MSc in applied mathematics from Brown University. Contact him at glin@ dam.brown.edu.

Xiaoliang Wan is a PhD candidate in the Division of Applied Mathematics at Brown University. His research interests include stochastic modeling and high-order numerical methods for partial differential equations. Contact him at xlwan@dam.brown.edu.

Chau-Hsing Su is a professor of applied mathematics at Brown University. His research interests include the study of spatial random processes and shock wave propagation. Su has a PhD in aeronautical engineering from Princeton University. Contact him at Chau-Hsing_Su@brown.edu.

George Em Karniadakis is a professor of applied mathematics at Brown University. His research interests include spectral methods on unstructured grids, parallel simulations of turbulence in complex geometries, and microfluidics simulations. Karniadakis has a PhD in mechanical engineering from MIT. Contact him at gk@ dam.brown.edu.

Writers

For detailed information on submitting articles,write to cise@computer.org or visit www.computer.org/cise/author.htm.

\section{Letters to the Editors}

Send letters to Jenny Stout, Lead Editor, jstout@computer.org.Provide an email address or daytime phone number with your letter.

\section{On the Web}

Access www.computer.org/cise/ or http://cise.aip.org for information.

\section{Subscribe}

Visit https://www.aip.org/forms/journal_catalog/order_form_fs.html or www.computer.org/subscribe/.

\section{Subscription Change of Address (IEEE/CS)}

Send change-of-address requests for magazine subscriptions to address.change@ieee.org. Be sure to specify CiSE.

\section{Subscription Change of Address (AIP)}

Send general subscription and refund inquiries to subs@aip.org.

\section{Missing or Damaged Copies}

Contact help@computer.org.For AIP subscribers, contact claims@aip.org.

\section{Reprints of Articles}

For price information or to order reprints, send email to cise@computer.org or fax +1 7148214010.

\section{Reprint Permission}

Contact William Hagen, IEEE Copyrights and Trademarks Manager, at copyrights@ieee.org.

www.computer.org/cise/ 